\title{
DESEMPENHO BALÍSTICO INDIVIDUAL DE COMPÓSITOS REFORÇADOS COM SISAL SOB MUNIÇÃO CALIBRE 7,62 MM*
}

\author{
Lucas Tedesco Bolzan ${ }^{1}$ \\ Luís Carlos da Silva ${ }^{2}$ \\ Sérgio Neves Monteiro ${ }^{3}$
}

\section{Resumo}

Nos dias contemporâneos, houve uma evolução do armamento utilizado e há uma tentativa dos centros de defesa e balística de acompanhar essa evolução no desenvolvimento de blindagens cada vez mais eficientes. Essa eficiência passa tanto na leveza do material quanto na sua capacidade de proteção. Vivemos também nos dias atuais uma preocupação com questões ambientais e energéticas e é justamente, envolto nesse cenário que pesquisas relacionadas à utilização de fibras naturais (FNL'S), tem se tornado cada vez mais relevantes. Desta forma, o objetivo deste trabalho é avaliar o desempenho individual de compósitos de matriz poliéster reforçados com fibras de sisal, quando submetidos ao impacto balístico de munição com calibre de 7,62 mm. Neste trabalho foram testados compósitos reforçados com $20 \%$ e $30 \%$ em volume de fibras de sisal. Com um auxílio de um radar Doppler, foram registradas as velocidades inicial (de impacto) e residual (após a perfuração do impacto). De posse desses dados foi possível calcular à energia cinética absorvida pelo compósito e estimar à velocidade limite do projétil que essa blindagem é capaz de suportar. Os resultados após alguns tratamentos estatísticos demonstraram que o compósito em questão tem resultados similares à outras blindagens reforçadas com FNL'S, representando mais uma opção de blindagem moderna.

Palavras-chave: Fibras naturais; Lignocelulósicas; Blindagem balística; Compósitos; Fibras de Sisal.

\section{INDIVIDUAL BALLISTIC PERFORMANCE OF COMPOSITES REINFORCED WITH SISAL UNDER CALIBER 7.62 MM AMMUNITION}

\section{Abstract}

In the contemporary days, there was an evolution of the armament used and there is an attempt of the centers of defense and ballistics to follow this evolution in the development of increasingly efficient armor. This efficiency passes on both the lightness of the material and its ability to protect. We are also currently concerned with environmental and energy issues, and it is precisely in this context that research related to the use of natural fibers (FNL'S) has become increasingly relevant. In this way, the objective of this work is to evaluate the individual performance of polyester matrix composites reinforced with sisal fibers, when submitted to ballistic impact of ammunition with a $7.62 \mathrm{~mm}$ caliber. In this work, composites reinforced with $20 \%$ and $30 \%$ by volume of sisal fibers were tested. With the aid of a Doppler radar, the initial velocities (impact) and residual velocities (after impact drilling) were recorded. With these data, it was possible to calculate the kinetic energy absorbed by the composite and to estimate the projectile velocity that the shield is capable of withstanding. The results after some statistical treatments showed that the composite in question has similar results to the other reinforcements reinforced with FNL'S, representing another option of modern shielding.

Keywords: Natural fibers; Lignocellulosic; Ballistic shielding; Composites; Sisal Fibers.

1 Engenheiro Civil, M.sc., Mestre em Ciência dos Materiais, Seção de Engenharia Mecânica e de Materiais, Instituto Militar de Engenharia, Rio de Janeiro, RJ Brasil.

2 Engenheiro Químico, D.Sc., Doutor em Ciência dos Materiais, Seção de Engenharia Mecânica e de Materiais, Instituto Militar de Engenharia, Rio de Janeiro, RJ, Brasil.

3 Engenheiro Metalurgista, Ph.D, Professor Titular, Seção de Engenharia Mecânica e de Materiais, Instituto Militar de Engenharia, Rio de Janeiro, RJ, Brasil. 


\section{INTRODUÇÃO}

A busca por estruturas de proteção balística tem sido interminável devido a evolução de armamentos cada vez mais sofisticados. As blindagens são estruturas materiais com a função de absorver ou dissipar a energia cinética oriunda do projétil. Diferentes materiais ou compósitos têm sido utilizados na blindagem balística, desde os tempos mais remotos até os dias atuais [1]. Atualmente ha um consenso de que um único material não pode ser utilizado como blindagem balística e sim um conjunto de materiais com funções específicas para absorver ou dissipar uma parte da energia cinética do projétil. Estes são os chamados sistemas de blindagens multicamadas (SBMs) associando diferentes materiais como cerâmicas, polímeros, metais e compósitos reforçados com fibras. Esses sistemas não visam apenas absorver a energia cinética do projétil, mas também impedir a penetração de fragmentos [2]. Os SBMs são geralmente compostos por uma camada frontal, de um material com elevada dureza, comumente um material cerâmico. A função dessa camada frontal é fraturar/erodir o projétil [3-5]. A dissipação de energia e consequentemente fragmentação desse projétil pela camada frontal se desenvolve através de mecanismos de nucleação, crescimento e coalescência de microtrincas [6]. A segunda camada, objeto desse estudo, pode ser escolhido como um material compósito, leve e capaz de absorver a energia cinética dos fragmentos projéteis e cerâmicos [7-8]. $E$ o material que forma a última camada pode ser escolhido como uma liga metálica de alumínio. Desde a última década a utilização de fibras naturais lignocelulósicas (FNL'S) em blindagens balísticas tem sido pesquisada em vários trabalhos científicos. Dentre as inúmeras vantagens podemos destacar o preço, a biodegradabilidade, a baixa massa específica e as suas propriedades mecânicas podem ser comparadas com as propriedades de outros tipos de fibras utilizadas como reforços [9]. As fibras de sisal provem da folha da planta Agave sisalana. Os principais produtores do sisal são o Brasil e a Tanzânia mas ele também é cultivado em alguns países da África, da América e do Extremo Oriente [10]. Os principais estudos a cerca das fibras de sisal estão relacionadas a propriedades mecânicas e estudos sobre a interface matriz e fibra em compósitos com matriz polimérica. Em estudo realizado por Martin e coautores [11], concluiu-se que a fibra de sisal é adequada para o uso em compósitos poliméricos, pois possui uma estabilidade térmica até aproximadamente $250^{\circ} \mathrm{C}$, além de outras aplicações [9].

\section{MATERIAIS E MÉTODOS}

As fibras de sisal foram adquiridas pela empresa Eletro Ferragens Santa Domênica em pacotes de $500 \mathrm{~g}$ e o tecido de sisal pela empresa Sisalsul - Fibras Naturais em um formato de rolo com as dimensões de $1,5 \mathrm{~m} \times 30 \mathrm{~m}$. A resina de poliéster cristal foi adquirida pela empresa Resinpoxy. As amostras foram moldadas em uma matriz metálica, cuja cavidade possui $119 \times 150 \times 10 \mathrm{~mm}$ e posterior compactação. Inicialmente é feita a limpeza e lubrificação da matriz com graxa de silicone, em seguida a mistura de poliéster e catalisador é colocada no molde metálico, onde são dispersas as fibras (ou tecido) de sisal, para que posteriormente seja realizada a prensagem uniaxial na prensa hidráulica SKAY com 5 toneladas de carga. O tempo de cura foi de aproximadamente 24 horas. A terceira e última camada testada consiste em uma fina chapa fabricada com liga de alumínio 5052 H34 de dimensões 120 x 150 x $4.76 \mathrm{~mm}$ adquirida pela empresa METALAK METAIS. Os compósitos de matriz de 
poliéster reforçados com $20 \%$ e $30 \%$ de sisal foram avaliados individualmente em ensaios de velocidade residual. Compósito com $20 \%$ e $30 \%$ em volume foram avaliados com duas amostras de cada. Cada corpo de prova foi submetido à 4 disparos. Os compósitos foram fixados em um suporte e com o auxílio do radar Doppler (FIG. 1), modelo SL-520P, do fabricante Weibel, foram registradas as velocidades do projétil antes $\left(\mathrm{V}_{0}\right)$ e após o impacto $\left(\mathrm{V}_{\mathrm{R}}\right)$, dessa forma foi possível calcular a energia cinética que foi absorvida pelos compósitos ( $E_{a b s)}$. Essa energia pode ser calculada pela seguinte expressão:

$$
E_{a b s}=\frac{m\left(V_{0}^{2}-V_{R}^{2}\right)}{2}(1)
$$

Onde: $\mathrm{m}=$ massa do projétil; $\mathrm{V}_{0}=$ velocidade de impacto do projétil na blindagem; $\mathrm{V}_{\mathrm{R}}$ $=$ velocidade residual do projétil após o impacto. $\mathrm{E}$ a sua velocidade limite $\left(V_{\mathrm{L}}\right)$ pode ser estimada pela seguinte expressão:

$$
V_{L}=\sqrt{\frac{2 \cdot E_{a b s}}{m}}(2)
$$

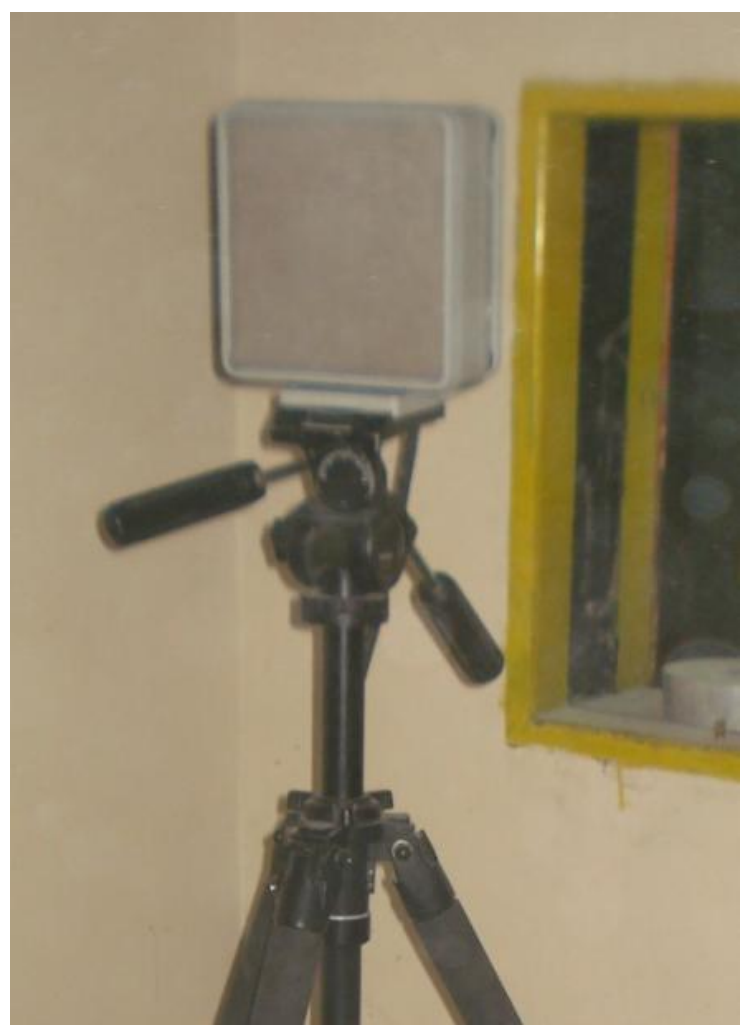

Figura 1. Radar Doppler, modelo SL-520P.

O ensaio balístico ocorreu utilizando uma munição comercial calibre $7,62 \mathrm{~mm}$, com distância ao alvo de 15 metros, da boca da arma até o ponto de contato com o material a ser atingido, conforme prevê a norma ABNT NBR 15000 para as munições 7,62 mm e um ângulo de incidência de 90 ․ A FIGURA 2 representa uma ilustração do esquema utilizado nesse ensaio. 


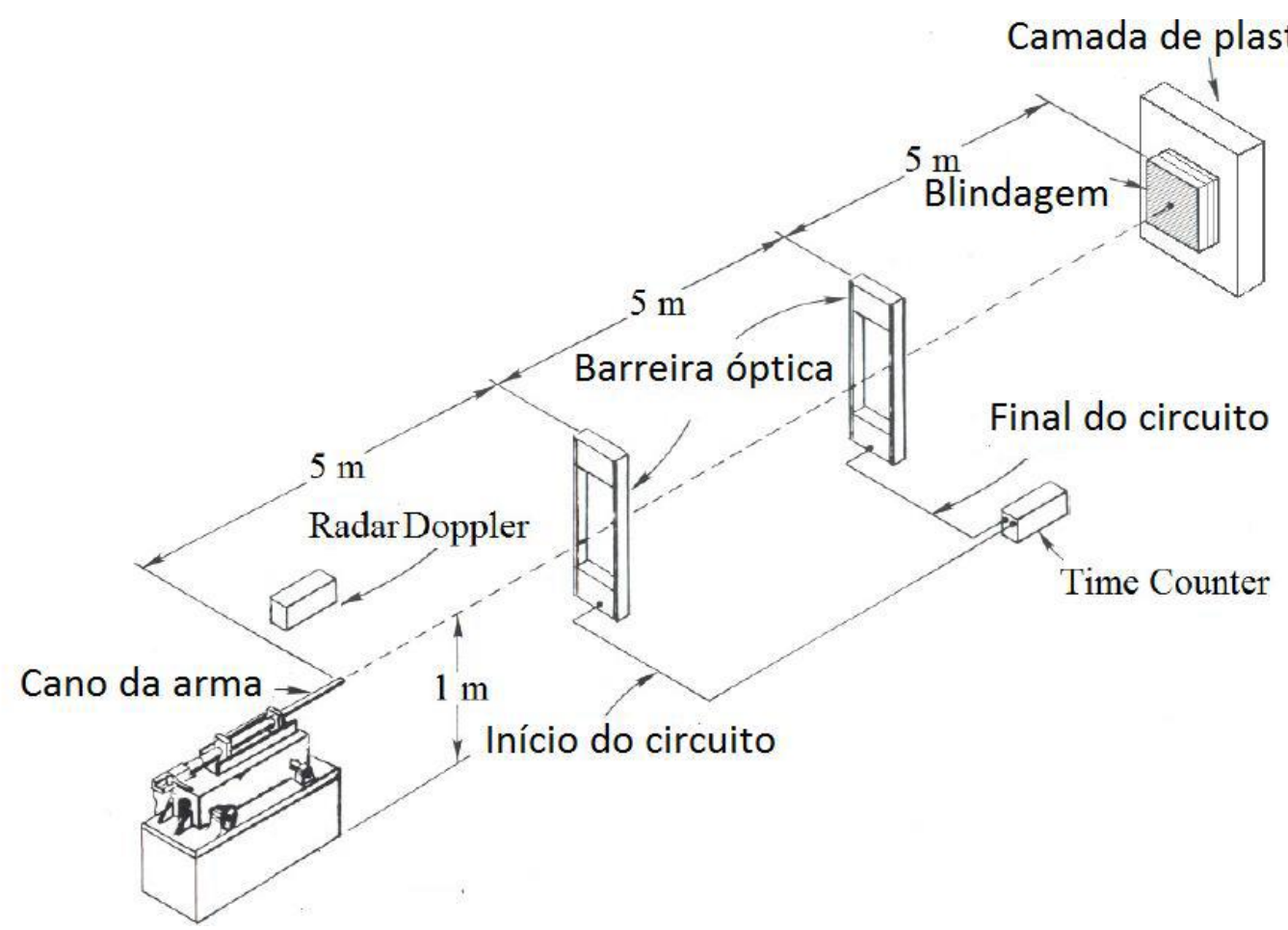

Figura 2. llustração do esquema para o ensaio balístico.

\section{RESULTADOS E DISCUSSÃO}

A TABELA 1 apresenta os resultados relacionados à energia absorvida de cada compósito submetidos ao impacto balístico de um projétil calibre $7,65 \mathrm{~mm}$.

TABELA 1. Energia absorvida e velocidade limite pelo material mediante impacto balístico

\begin{tabular}{cccccc}
\hline $\begin{array}{c}\text { Componente } \\
\text { da } \\
\text { blindagem }\end{array}$ & $\mathbf{V}_{\mathbf{0}}(\mathbf{m} / \mathbf{s})$ & $\mathbf{V}_{\mathbf{R}}(\mathbf{m} / \mathbf{s})$ & $\mathrm{E}_{\mathrm{ABS}}(\mathbf{J})$ & $\% \mathrm{E}_{\mathrm{ABS}}$ & $\mathbf{V}_{\mathrm{L}}(\mathbf{m} / \mathbf{s})$ \\
\hline $\begin{array}{c}\text { Poliéster - } \\
\text { Sisal - 30\% } \\
\text { Tecido }\end{array}$ & $845 \pm 5$ & $828 \pm 5$ & 139 & 4,0 & $170 \pm 3$ \\
\hline $\begin{array}{c}\text { Poliéster - } \\
\text { Sisal - 30\% } \\
\quad \text { Fibras }\end{array}$ & $845 \pm 6$ & $828 \pm 6$ & 139 & 4,0 & $169 \pm 9$ \\
\hline $\begin{array}{c}\text { Poliéster - } \\
\text { Sisal - 20\% } \\
\text { Tecido }\end{array}$ & $834 \pm 6$ & $816 \pm 6$ & 143 & 4,2 & $172 \pm 9$ \\
\hline $\begin{array}{c}\text { Poliéster - } \\
\text { Sisal - 20\% } \\
\text { Fibras }\end{array}$ & $834 \pm 13$ & $819 \pm 13$ & 116 & 3,4 & $155 \pm 7$ \\
\hline
\end{tabular}

Através do método de Weibull utilizou-se uma análise estatística para estes dados. Os gráficos da FIGURA 3 demonstra a distribuição de Weibull das velocidades limites 
para os compósitos contendo $20 \%$ e $30 \%$ de fibras e tecido. A TABELA 2 apresenta os parâmetros de Weibull.

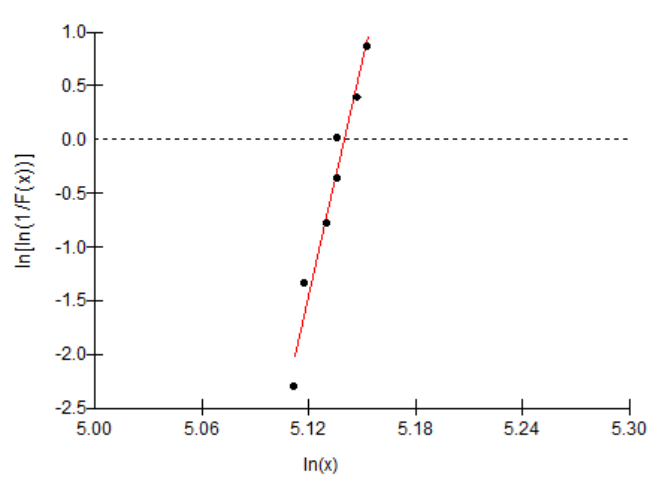

(a)

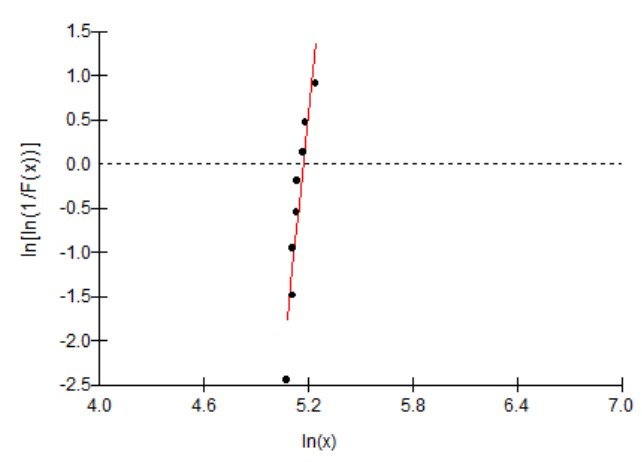

(c)

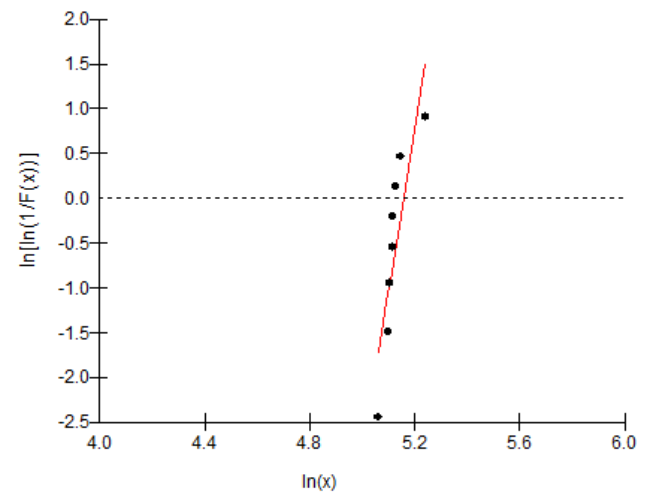

(b)

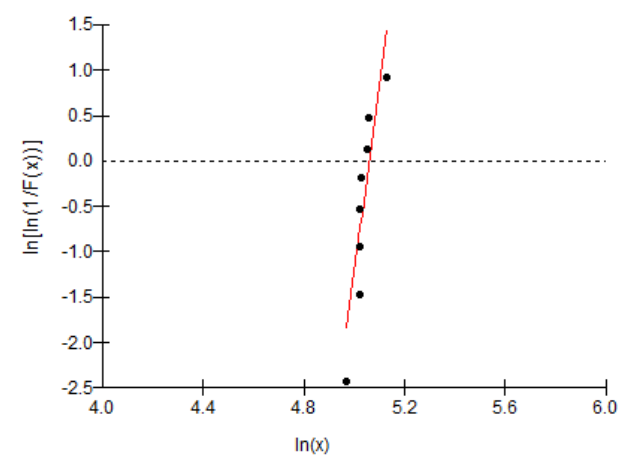

(d)

FIGURA 3. Gráfico da distribuição de Weibull das velocidades limites para a blindagem com compósito de poliéster reforçado com: a) 30\% de tecido de sisal; b) $30 \%$ de fibras de sisal; c) $20 \%$ de tecido de sisal; d) $20 \%$ de fibras de sisal.

TABELA 2. Parámetros de Weibull para velocidade limite do compósito poliéster sisal

$\begin{array}{cccc}\text { Camada } & \text { Módulo de } & \text { Unidade } & \text { Coeficiente de } \\ \text { Intermediária } & \text { Weibull }(\beta) & \begin{array}{c}\text { característica } \\ \text { correlação } \mathbf{R}^{2}\end{array}\end{array}$

(७)

\begin{tabular}{llll}
\hline $\mathbf{3 0} \%$. Tecido & 72,05 & 170,7 & 0,9679 \\
\hline $\mathbf{3 0}$. Fibras & 18,04 & 173,8 & 0,7391 \\
\hline $\mathbf{2 0} \%$. Tecido & 18,70 & 175,7 & 0,8633 \\
\hline $\mathbf{2 0} \%$. Fibras & 20,46 & 157,5 & 0,8484 \\
\hline
\end{tabular}

Os dados da tabela 2 demonstram que os dados referentes ao compósito reforçado com $30 \%$ de tecido apresentam a maior homogeneidade $(\beta=72,05)$, além de representar também o melhor ajuste $\left(\mathrm{R}^{2}=\right.$ 0,9679 ) já o maior valor de $\theta$, que nesse caso representa a velocidade limite que melhor caracteriza a amostra, está associado às amostras fabricadas com $20 \%$ de tecido de sisal $(\theta=175,7)$. 


\section{CONCLUSÃO}

Os compósitos testados individualmente neste trabalho, contra munições de calibre $7,62 \mathrm{~mm}$, não apresentaram diferenças significativas no seu comportamento balístico, visto que os valores de velocidade limite desses materiais foram próximos. Os valores apresentados são bastante próximos de outras fibras naturais e pode representar mais uma possibilidade viável de material destinado a blindagem balística, além desses SBMs com camada intermediária de compósitos de poliéster-sisal serem significativamente mais baratos do que aqueles que contém laminado de aramida, proporcionando uma redução de custo de $38 \%$.

\section{REFERÊNCIAS}

1 SERJOUEI, A.; CHI, R.; ZHANG, Z.; SRIDHAR, I. Experimental Validation of BLV Model on Bi-Layer Ceramic-Metal Armor. International Journal of Impact Engineering, Vol. 77, p. 30-41. 2015.

2 A.S. Brown; High Perform. Compos., Vol 1, pp. 23-26. 2003.

3 C.E. Anderson and B.L. Morris: Int. J. Impact Eng., 1992, vol. 12, pp. 167-87.

4 M.M. Shokrieh and G.H. Javadpour: Compos. Struct., 2008, vol. 82, pp. 269-76.

5 E. Medvedovski: Ceram. Int., 2010, vol. 36, pp. 2103-15, 2117-27.

6 L.H.L. Louro and M.A. Meyers: J. Mater. Sci., 1989, vol. 24, pp. 2516-32.

7 S.C. Chou, E. De Luca, J. Pritti, and W. Betheney: Compos. Sci. Technol., 1998, vol. 58, pp. 1453-61.

8 J.R.M. d'Almeida, L.M. Nunes, and S. Paciornik: Compos. Sci. Technol., 2004, vol. 64, pp. 945-54.

9 L.T., Bolzan, Avaliação do Comportamento Balístico de um Sistema de Blindagem Multicamada com Compósito de Poliéster Reforçado com Fibra e Tecido de Sisal, Dissertação de mestrado do curso de Pós-Graduação em Ciência dos Materiais no Instituto Militar de Engenharia, 2016.

10 MOHANTY, A. K.; MISRA, M.; DRZAL, L. T. Natural Fibers, Biopolymers, and Biocomposites. Taylor and Francis, New York, 2005.

11 MARTIN, A. R.; MARTINS, M. A.; MATTOSO, L. H. C.; SILVA, O. R. R. F. Caracterização química e estrutural de fibra de sisal da variedade Agave sisalana. Polímeros, Vol. 19, n. 1, p. 40-46, 2009. 